\title{
Constraining dark energy interacting models with WMAP
}

\author{
Germán Olivares*, Fernando Atrio-Barandela ${ }^{\dagger}$ and Diego Pavón* \\ * Departamento de Física, Universidad Autónoma de Barcelona, Spain \\ ${ }^{\dagger}$ Departamento de Física Teórica, Universidad de Salamanca, Spain
}

\begin{abstract}
We determine the range of parameter space of an interacting quintessence (IQ) model that best fits the luminosity distance of type Ia supernovae data and the recent WMAP measurements of Cosmic Microwave Background temperature anisotropies. Models in which quintessence decays into dark matter provide a clean explanation for the coincidence problem. We focus on cosmological models of zero spatial curvature. We show that if the dark energy (DE) decays into cold dark matter $(\mathrm{CDM})$ at a rate that brings the ratio of matter to dark energy constant at late times, the supernovae data are not sufficient to constrain the interaction parameter. On the contrary, WMAP data constrain it to be smaller than $c^{2}<10^{-2}$ at the $3 \sigma$ level. Accurate measurements of the Hubble constant and the dark energy density, independent of the CMB data, would support/disprove this set of models.
\end{abstract}

\section{INTRODUCTION}

Recent observational data suggest the Universe has entered a period of accelerated expansion [1]. The $\Lambda \mathrm{CDM}$ model is the simplest model that best fits the supernovae luminosity distance and CMB anisotropy spectrum data [1,2]. Though it looks a very serious candidate it raises two main theoretical problems. First, it assumes the energy density of the vacuum but according to quantum field theory it should be larger than observed by 120 orders of magnitude. Secondly, there is the coincidence problem, namely: why the energy densities of matter and dark energy -scaling so differently with expansion- happen to be of the same order precisely today? To solve the latter problem models featuring an interaction between quintessence and cold dark matter has been proposed $[3,4]$.

\section{THE INTERACTING QUINTESSENCE MODEL}

To account for the coincidence problem Zimdahl et al. [3] postulated an interaction between cold dark matter and dark energy (a quintessence scalar field).

$$
\dot{\rho}_{x}+3 H\left(1+w_{x}\right) \rho_{x}=-\delta, \quad \dot{\rho}_{c d m}+3 H \rho_{c d m}=\delta .
$$

This brings the ratio between their energy densities, $r \equiv \rho_{c d m} / \rho_{x}$, to a stable constant value at late times. The interaction term, $\delta$, may depend on $H, \rho_{x}$ and $\rho_{c d m}$. A simple ansatz is $\delta=3 H c^{2}\left(\rho_{x}+\rho_{c d m}\right)$, where the constant $c^{2}$ is the interaction parameter. From 


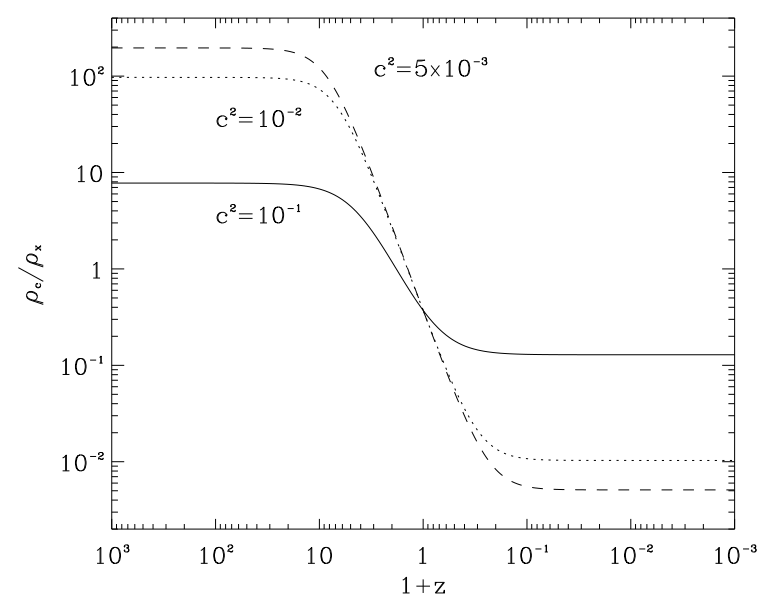

FIGURE 1. Evolution of the energy density ratio $r=\rho_{c d m} / \rho_{x}$ from an unstable maximum towards a stable minimum (at late times) for different values of $c^{2}$. As current value we take $r_{0}=0.42$.

Eqs. (1) the aforesaid ratio is constrained to evolve between two stationary values, $r_{ \pm}=-\frac{1+w_{x}}{c^{2}} \pm \sqrt{\frac{w_{x}^{2}}{4 c^{4}}+\frac{w_{x}}{c^{2}}}$, where $r_{+}$denotes the unstable value and $r_{-}$the stable one -see Ref. [4] for details. As seen from Fig. 1 the stronger the interaction, the smaller $\rho_{c d m}$ in the past. Obviously, such a dissimilar evolution of $\rho_{c d m}$ as compared to the $\Lambda C D M$ model, gives rise to a different expansion rate and a different evolution of the metric perturbations. The former modifies the luminosity distance of SNIa. The latter should be observed in the CMB anisotropy spectrum. Below we constrain the IQ model with the SNIa data of Riess et al. [1] and the CMB anisotropy data collected by the WMAP satellite [2].

\section{OBSERVATIONAL CONSTRAINTS}

To constrain the IQ model with the SNIa data we assume the following priors: a spatially flat model $\left(\Omega_{k}=0\right)$ and $-1<w_{x}<-0.6$. As seen from Fig. 2 the lack of data for redshifts $z>1.8$ does not constrain $c^{2}[5]$.

To constrain the model with the WMAP data we adopt a phenomenological point of view and impose that the interaction exists along the streamlines of the fluids (dark energy and CDM), i.e.,

$$
u^{\nu} \nabla_{\mu} T_{v(c d m)}^{\mu}=-u^{\mu} \nabla_{\mu} \rho_{c d m}-3 H\left(\rho_{c d m}+P_{c d m}\right)=-\delta
$$

The net momentum transfer between CDM and dark energy alongside the accelerated expansion, limits the growth of the CDM perturbations. We implement the perturbations 


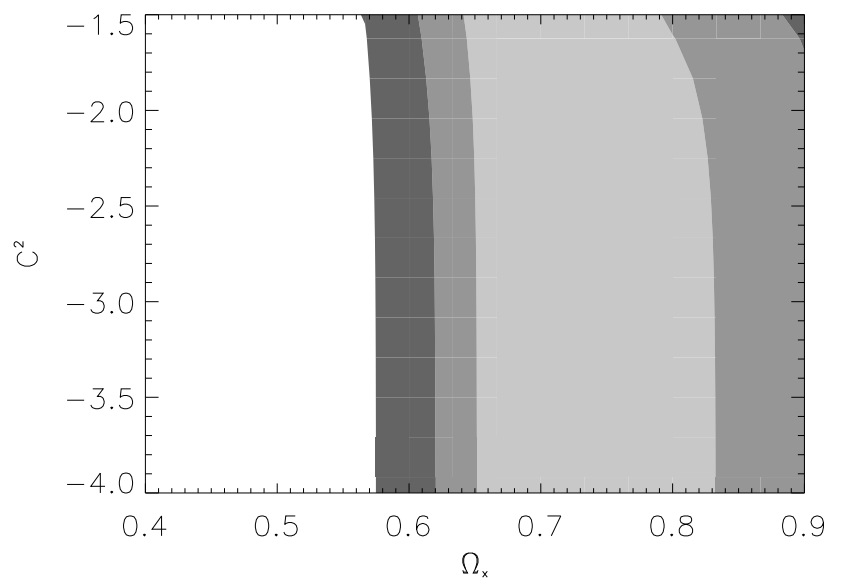

FIGURE 2. Joint confidence intervals at $68 \%, 95 \%$ and $99.9 \%$ C.L. of the IQ model fitted to the "gold" sample of SNIa data of Riess et al. [1]. For convenience, the $c^{2}$ axis is represented using a logarithmic scale and it has been cut to $c^{2} \leq 10^{-4}$, though models with $c^{2}=0$ have been included in the analysis.

TABLE 1. Mean values and $1 \sigma$ C.L. of the parameters of the IQ and $\Lambda$ CDM models.

\begin{tabular}{lcc}
\hline \multicolumn{1}{c}{ Parameter } & IQ model & $\Lambda$ CDM model \\
\hline Interaction parameter, $c^{2}$ & $<5 \times 10^{-3 \dagger}$ & $0^{* *}$ \\
CDM density/critical density, $\Omega_{c}$ & $0.4 \pm 0.1$ & $0.29 \pm 0.07$ \\
Baryon density/critical density, $\Omega_{b}$ & $0.063 \pm 0.012$ & $0.047 \pm 0.006$ \\
Hubble constant, $h_{0}$ & $0.61 \pm 0.06$ & $0.72 \pm 0.05$ \\
Spectral tilt, $n_{s}$ & $0.96 \pm 0.03$ & $0.99 \pm 0.04$ \\
Amplitude, $A$ & $0.9 \pm 0.1$ & $0.9 \pm 0.1$ \\
\hline * $A M A P$ team results $[2]$. & & \\
$\dagger$ Upper limit at $1 \sigma$ C.L. & & \\
$* *$ & & \\
This is a prior of the $\Lambda$ CDM model. & &
\end{tabular}

equations (2) together with $\nabla_{\mu} T_{v(x)}^{\mu}=-\nabla_{\mu} T_{\nu(c d m)}^{\mu}$ in the CMBfast code [6] and assume a flat model with $\left(\Omega_{k}=0\right)$, universes older than 12 Gyrs, BBN, and $-1<w_{x}<0.6$. By means of a Monte Carlo Markov chain we reconstruct the surface of the likelihood in the 7D-space parameter defined by the cosmological parameters $c^{2}, \Omega_{x}, w_{x}, \Omega_{b}, H_{0}, n_{s}, A$. Table 1 summarizes our results. Due to the interaction the CDM redshift is smaller than in the $\Lambda C D M$ model, therefore a higher value of $\Omega_{c d m}$ is needed to have enough gravitational potential at matter-radiation decoupling. Moreover, in order not to alter the height of the acoustic peaks the $\Omega_{b}$ value increases. Otherwise, the small mean value of $H_{0}$ would suppress them. The likelihood shows clearly that the interaction parameter $c^{2}$ is compatible with the WMAP data (see Fig. 3). Nevertheless, non-interacting models are also allowed, unless the amplitude, $A$, is not a parameter (see Ref. [5]). Thus, $c^{2}$ is bounded from above. At $1 \sigma$ confidence level $c^{2}<5 \times 10^{-3}$. The spectral tilt, $n_{s}$, and the amplitude of the perturbations at the horizon size, $A$, are in perfect agreement with those 

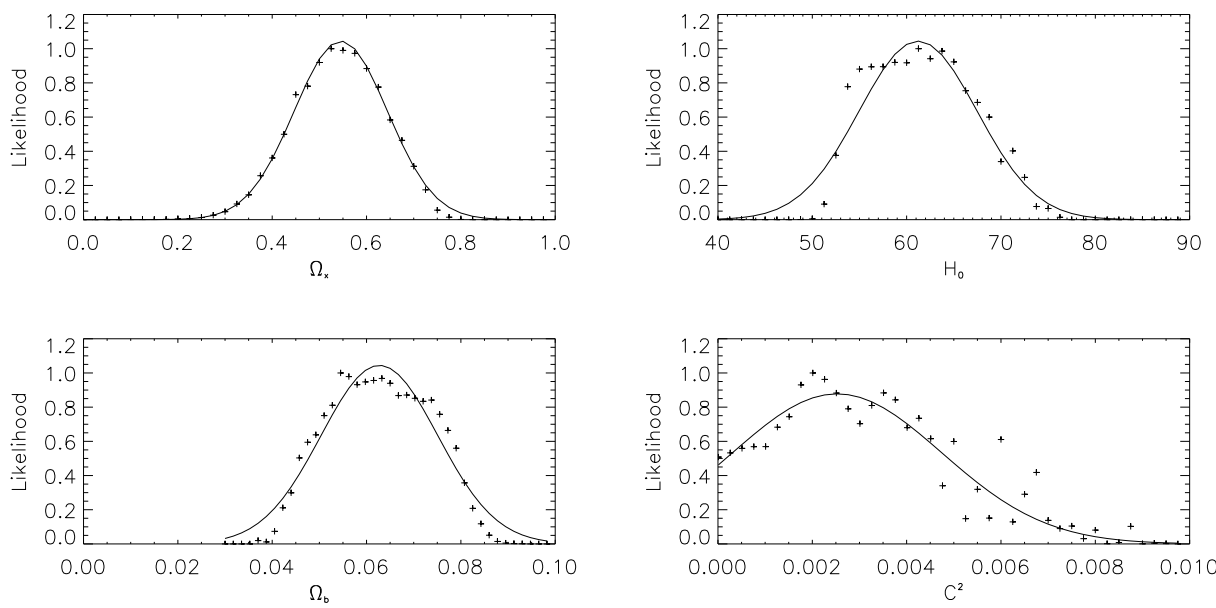

FIGURE 3. Crosses represent the likelihood computed from 50,000 models, marginalized over all parameters but one. The solid line represents the best Gaussian fit.

of the WMAP team [2].

\section{CONCLUSIONS}

We have presented an interacting cosmological model that solves the coincidence problem. Despite the seemingly odd results of Tab. 1 it is in perfect agreement with SNIa data [1] and CMB data [2]. Moreover, the lack of power at the lower multipoles becomes a less serious problem. Indeed, because of the smaller redshift evolution of CDM energy density the gravitational potential evolves slower than in the $\Lambda$ CDM model. Thus, a smaller Integrated Sachs-Wolfe effect produces less power in the CMB anisotropy spectrum. A next generation of accurate measurements of the Hubble parameter and the dark energy density, independent of the CMB data, are needed to support/disprove this kind of models.

\section{ACKNOWLEDGMENTS}

We thank the organizers of the XXVIIIth edition of the "Encuentros Relativistas Españoles" for this opportunity. This research was partly supported by the Spanish Ministry of Science and Technology under Grants BFM2003-06033, BFM2000-1322 and the Junta de Castilla y León (projects SA002/03, SA010C05).

\section{REFERENCES}

1. A.G. Riess et al., Astrophys. J., 607, 665 (2004). 
2. D.N. Spergel et al., Astrophys. J. Suppl. Ser., 148, 175 (2003).

3. W. Zimdahl, D. Pavón, and L.P. Chimento, Phys. Lett. B, 521, 133 (2001).

4. L.P. Chimento, A.S. Jakubi, D. Pavón, and W. Zimdahl, Phys. Rev. D, 67, 083513 (2003).

5. G. Olivares, F. Atrio-Barandela, and D. Pavón, Phys. Rev. D, 71, 063523 (2005).

6. U. Seljak, and M. Zaldarriaga, Astrophys. J., 469, 437 (1996). See http://www.cmbfast.org 